\title{
DEUX CUMACES NOUVEAUX DE L'ATLANTIQUE TROPICAL: ATLANTISTYLIS CHAUVINI N. G., N. SP. (DIASTYLIDAE) ET PSEUDODIASTYLIS DELAMAREI N. SP. (LAMPROPIDAE) ${ }^{1}$ )
}

\author{
PAR \\ DANIEL REYSS \\ Centre Océanologique de Bretagne, B.P. 337, 29273 Brest, France
}

- L'étude d'une collection de Cumacés (Diastylidae et Lampropidae) provenant de la campagne 31 du N.O. „Atlantis" II (Woods Hole Oceanographic Institution) entre Dakar et Recife en février 1967, a fourni un certain nombre d'individus appartenant, soit à un genre nouveau, soit à une espèce nouvelle d'un genre jusqu'ici monospécifique et connu d'après un seul exemplaire.-

\section{Atlantistylis n. g. ${ }^{2}$ )}

Diagnose. - Diastylidae caractérisé par un telson très court (plus court que le dernier segment) garni de deux épines apicales et sans épines latérales.

Mâle: pas de pléopodes, les exopodes des MXP3, P1-P4 avec le premier article dilaté. Rame interne des uropodes à deux articles. Antennes plus courtes que le corps, atteignant le 3ème segment abdominal.

Femelle: des exopodes rudimentaires sur les P3 et les P4, des exopodes (sans premier article dilaté) sur les PMX3, P1 et P2. Rame interne de l'uropode à 3 articles.

Matériel étudié.

\section{Atlantistylis chauvini n. sp. ${ }^{3}$ )}

Station $142\left(10^{\circ} 30^{\prime} \mathrm{N} 17^{\circ} 51^{\prime} \mathrm{W}\right), 1624 \mathrm{~m}$ : 14 exemplaires (dont l'holotype, of de $4 \mathrm{~mm}$ ).

Station $155\left(00^{\circ} 03^{\prime} \mathrm{S} 27^{\circ} 48^{\prime} \mathrm{W}\right), 3730 \mathrm{~m}$ : 1 exemplaite.

Station $156\left(00^{\circ} 46^{\prime} \mathrm{S} 29^{\circ} 28^{\prime} \mathrm{W}\right), 3459 \mathrm{~m}: 1$ exemplaire.

Station $167\left(07^{\circ} 58^{\prime} \mathrm{S} 34^{\circ} 17^{\prime} \mathrm{W}\right), 943 \mathrm{~m}$ : 1 exemplaire.

Station $169\left(08^{\circ} 03^{\prime} \mathrm{S} 34^{\circ} 23^{\prime} \mathrm{W}\right), 587 \mathrm{~m}$ : 1 exemplaire.

Description. - Mâle. Carapace, forme d'ovale allongé, égale au 1/4 de la longueur totale de l'animal. Pseudorostre pointu, long, égal au $1 / 3$ de la carapace.

L'encoche antennaire est bien marquée. Les téguments sont minces, peu calcifiés

1) Contribution $n^{\circ} 161$ du Département Scientifique du Centre Océanologique de Bretagne.

2) En l'honneur de l'Atlantis II de la W.H.O.I.

3) Cette espèce est respectueusement dédiée au Commandant $\mathbf{R}$. Chauvin, premier directeur du Centre Océanologique de Bretagne. 


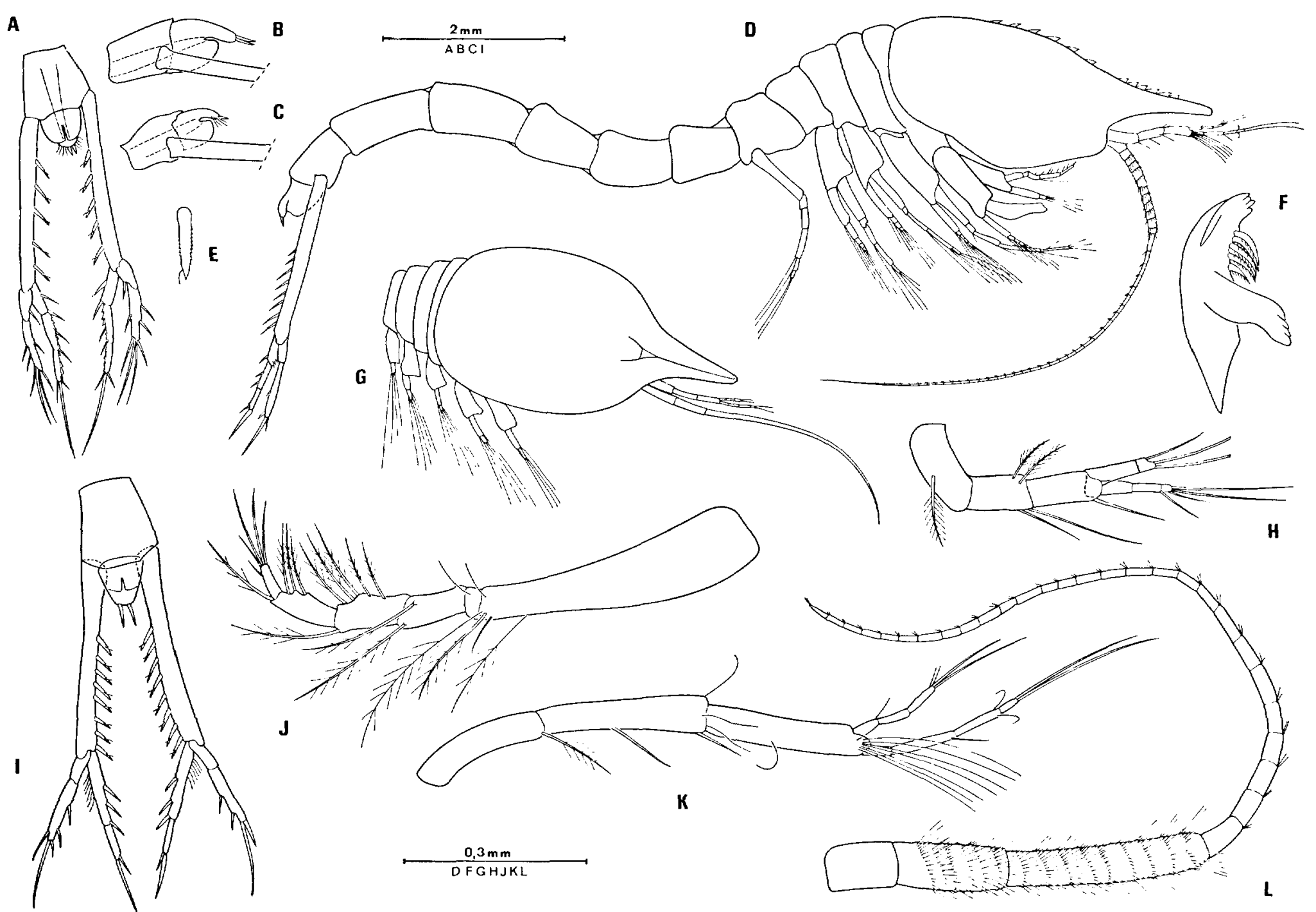

Fig. 1. Atlantistylis cbauvini n. g., n. sp. A, telson $q ; \mathrm{B}$, telson $\hat{\delta} ; \mathrm{C}$, telson $q ; \mathrm{D}$, habitus $\hat{\delta}$;

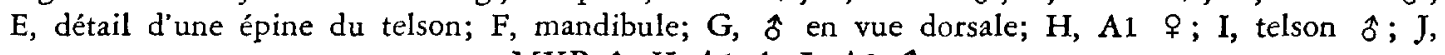
MXP $\hat{\sigma} ; \mathrm{K}, \mathrm{A} 1 \hat{\delta} ; \mathrm{L}, \mathrm{A} 2 \hat{\delta}$. 
et transparents. Il existe une fine rangée de denticulations dirigées vers l'avant sur la ligne médio-dorsale de la carapace et du pseudorostre.

Il n'existe pas de denticulations au bord ventral de la carapace (fig. 1D, 1G).

Les segments thoraciques sont bien développés, tous visibles dorsalement, sans ornementation particulière.

L'abdomen est long; les trois premiers segments sont de taille semblable et plus courts que les segments 4 et 5 . Le dernier segment est le plus court de tous. Tous ces segments sont sans ornementation (fig. 1D).

Le telson est plus court que le dernier segment abdominal, la partie postanale porte deux épines apicales et il n'y a aucune épine latérale (fig. 1I, 1B).

Les segments thoraciques sont bien développés, tous visibles dorsalement, sans ornementation particulière.

L'abdomen est long; les trois premiers segments sont de taille semblable et plus courts que les segments 4 et 5 . Le dernier segment est le plus court de tous. Tous ces segments sont sans ornementation (fig. 1D).

Le telson est plus court que le dernier segment abdominal, la partie postanale porte deux épines apicales et il n'y a aucune épine latérale (fig. 1I, 1B).

L'antennule est bien développée, avec un pédoncule à trois articles de même taille dont le dernier porte les flagelles et un faisceau de longues soies. Le flagelle principal possède 4 articles et le flagelle accessoire est aussi long que les $2 / 3 \mathrm{du}$ flagelle principal (fig. $1 \mathrm{~K}$ ).

L'antenne a un pédoncule portant des rangées de courtes soies disposées en spirale et un flagelle multi-articulé avec deux soies à la base de chacun de ses articles. L'antenne est plus courte que le corps et atteint, sans le dépasser, le 3ème segment abdominal (fig. 1L).

Les pièces buccales sont banales pour un Diastylide; la mandibule (fig. 1F) possède une base pointue et un processus molaire légèrement oblique et incliné vers la base.

Les MXP2 possèdent un basis aussi long que l'ensemble des autres articles (fig. 1J).

Les MXP3 ont un basis bien développé mais non dilaté. L'exopodite possède un premier article large, aplati en palette, 3 à 4 fois plus large que le second article (fig. 2A).

Les pattes thoraciques sont assez courtes, robuste et sans ornementation particulière. Les exopodites des $\mathrm{P} 1, \mathrm{P} 2, \mathrm{P} 3$ et $\mathrm{P} 4$ ont tous, comme ceux des MXP3, un premier article dilaté en palette (fig. $2 \mathrm{~B}, 2 \mathrm{E}, 2 \mathrm{G}, 2 \mathrm{I}$ et $2 \mathrm{~K}$ ).

Aucun des exemplaires observés ne possède de pléopodes, même rudimentaire.

Le pédoncule des uropodes est plus court que l'ensemble des trois derniers segments abdominaux. Il porte, sur son bord interne, 8 épines flagellées et finement denticulées (fig. 1I, 1E).

Les deux rames de l'uropode ont sensiblement la même taille, l'interne étant légèrement plus longue que l'externe. La rame interne possède deux articles, le premier deux fois plus long que le second et portant 3 épines au bord interne et 
CUMACÉS DE L'ATLANTIQUE

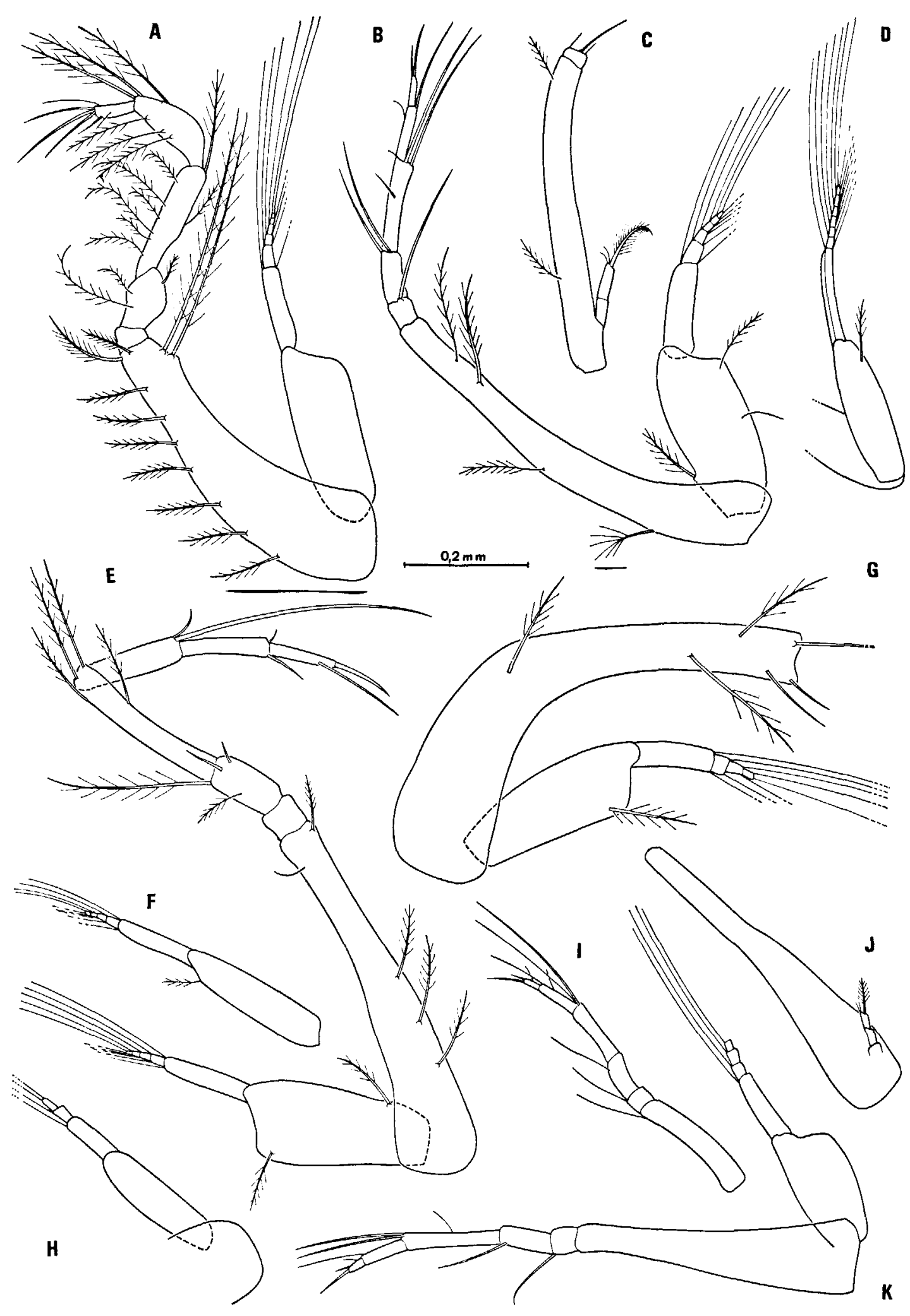

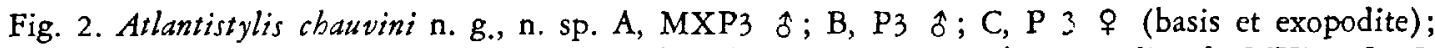

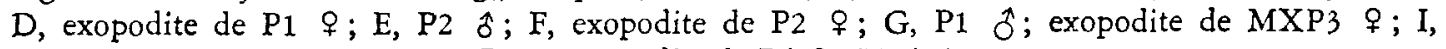
P5; J, exopodite de P4 $q ; \mathrm{K}, 4$ of. 
quelques soies au bord externe. Le second article, court, porte une épine au bord interne distal et une longue épine apicale (plus longue que l'article). La rame externe possède un premier article court, inerme, et un second article deux fois plus long que le premier, portant deux épines internes, une épine au bord distal externe et une très longue épine apicale, aussi longue que le second article (fig. 1I).

Femelle. La forme générale du corps est la même que chez le mâle.

Le telson est légèrement plus court chez la femelle, en particulier dans sa partie postanale où il existe deux épines apicales et quelques paires de soies latérales, mais pas d'épine latérale.

Le pédoncule des uropodes porte, sur son bord interne, 6 épines finement denticulées et flagellées.

La rame interne de l'uropode possède trois articles (deux seulement chez le mâle) de taille semblable. Les deux premiers portent chacun deux épines au bord interne et le troisième possède une épine au bord distal interne, une soie au bord distal externe et une très longue et fine épine apicale, égale à la longueur des deux derniers articles de la rame. On peut observer, en outre, sur le bord interne des deux derniers articles, entre les épines, une fine rangée de denticulations (fig. $1 \mathrm{~A}$ et $1 \mathrm{C}$ ).

La rame externe, plus courte que l'interne, possède deux articles; le premier, court, porte une épine au bord interne et le second, deux fois plus long que le premier, une épine sur le bord interne, deux épines sur le bord externe, une épine au bord distal externe, une épine plus longue que la précédente au bord distal interne et deux longues épines apicales (fig. 1A).

L'antennule est courte, avec un pédoncule à trois articles de même taille, un flagelle principal, également à trois articles dont la taille totale est celle d'un des articles du pédoncule (deux longs et un terminal très court) et un flagelle accessoire à deux articles, presqu'aussi long que le flagelle principal (fig. $1 \mathrm{H}$ ).

Les pièces buccales sont semblables dans les deux sexes. Les PMX3, P1 et P2 possèdent des exopodites de taille normale, mais à la différence des exopodites correspondants du mâle, le premier article n'est pas aplati en palette (fig. 2F, $2 \mathrm{H}$ et $2 \mathrm{D}$ ).

Les $\mathrm{P} 3$ et $\mathrm{P} 4$ portent des exopodites rudimentaires, composés de deux courts articles de taille semblable et dont le second porte, à son extrémité, deux courtes soies et une longue et épaisse soie plumeuse (fig. 2G, 2J).

Discussion. - Nous avons utilisé, pour cette discussion, la clé des genres de Diastylidae établie par Jones (1969).

On peut distinguer, d'après cette clé, deux groupes de Diastylidae caractérisés par la présence ou l'absence de pléopodes chez le mâle.

L'état de développement des exopodes des pattes thoraciques (P1-P4) particulièrement bien adaptés à la nage, la longueur de l'antenne permettent d'affir- 
mer que les mâles d'Atlantistylis que nous avons observés étaient adultes. Or aucun ne portait de pléopodes. Quand l'on sait que les pléopodes apparaissent sous forme rudimentaire lors des stades pré-adultes, stades pendant lesquels les exopodes des pattes thoraciques et les antennes n'ont pas encore atteint leur plein développement, on peut dire que l'absence de pléopodes chez le mâle d'Atlantistylis est bien un caractère générique.

Cependant comme il est toujours délicat de discuter de la validité d'un genre à partir d'un seul caractère sexuel, nous comparerons successivement Atlantistylis aux différents genres de Diastylidae des deux groupes définis par la présence ou l'absence de pléopodes chez le mâle.

a) Parmi les différents genres de Diastylidae possédant des pléopodes chez les mâles, seul le genre Leptostylis Sars, 1869, présente un ensemble de caractères communs avec Atlantistylis n. g. En effet, ces deux genres ont un telson court, des mandibules normales (non tronquées à la base), peu ou pas d'épines latérales sur la partie post-anale du telson, l'exopode de l'uropode plus court que l'endopode et l'antenne du mâle plus courte que le corps.

Le genre Leptostylis diffère cependant du genre Atlantistylis par les caractères suivants:

Le flagelle accessoire de l'antennule du mâle et de la femelle d'Atlantistylis est beaucoup plus développé que chez Leptostylis où il ne dépasse pas la moitié du flagelle principal chez le mâle et la longueur du premier article du flagelle principal chez la femelle.

L'antennule du mâle de Leptostylis a un pédoncule court, dont le dernier segment à extrémité hémisphérique porte un large pinceau de soies cachant le flagelle (ce pinceau de soies manque cependant souvent sur des spécimens abimés). Or la structure générale de l'antennule d'Atlantistylis est différente.

Il existe des pléopodes rudimentaires chez les mâles immatures de Leptostylis et des pléopodes normaux chez l'adulte. Chez Atlantistylis nous n'en avons jamais observé, même rudimentaire.

Le telson de Leptostylis est de taille égale à celle du dernier pléonite et porte 1 à 3 paires d'épines latérales (Bacescu-Mester, 1967). En fait, seul L. producta Norman, 1879, connu à un seul exemplaire, ne possèderait pas d'épines latérales. Toutes les autres espèces du genre en possèdent.

L'antenne du mâle ne dépasse pas les segments thoraciques chez Leptostylis alors qu'elle atteint chez Atlantistylis le troisième segment abdominal.

Enfin, si la rame interne de l'uropode est toujours plus courte que l'externe (ou de même longueur), elle est tri-articulée chez le mâle de Leptostylis et bi-articulée chez le mâle d'Atlantistylis.

Il ne peut donc pas y avoir de confusion possible entre ces deux genres.

b) Si l'on admet maintenant, comme cela semble être le cas, que les mâles d'Atlantistylis sont dépourvus de pléopodes, seul le genre Dic Stebbing, 1910, possède comme Atlantistylis, des exopodites sur les MXP3 de la femelle. Les 
deux genres diffèrent cependant en particulier par la grande longueur du telson et l'ischium très développé des MXP3 chez Dic.

Parmi les genres dont les PMX3 sont dépourvus d'exopodite, le genre Gynodiastylis Calman, 1911, possède un telson assez semblable à celui d'Atlantistylis, souvent très court et parfois dépourvu d'épines latérales. Notons que les 37 espèces de Gynodiastylis proviennent toutes de l'Océan Pacifique et de l'Océan Indien, et qu'aucune n'a été trouvée à plus de $150 \mathrm{~m}$ de profondeur. Il ne semble donc pas qu'il puisse y avoir de confusion possible entre Atlantistylis n. g. et Dic ou Gynodiastylis.

Matériel étudié.

\section{Pseudodiastylis delamarei n. sp. ${ }^{4}$ )}

Station $167\left(07^{\circ} 58^{\prime} \mathrm{S} 34^{\circ} 13^{\prime} \mathrm{W}\right), 943-1007 \mathrm{~m}: 13$ exemplaires dont l'holotype ( ô de $5 \mathrm{~mm}$ ).

Station $159\left(07^{\circ} 58^{\prime} \mathrm{S} 34^{\circ} 22^{\prime} \mathrm{W}\right), 834-939 \mathrm{~m}$ : 1 exemplaire.

Description. - Mâle. Le corps est court et massif et l'allure générale de la carapace, avec ses carènes, rappelle Campylaspis vitrea.

Le pseudorostre est pointu, bien marqué et n'est pas redressé vers le haut. Il est orné de quelques petites dents.

La carapace porte une série de carènes: latéralement on peut observer une courte carène antérieure, transversale et médiodorsale. Plus postérieurement, une carène complète, partant du bord ventral de l'encoche antennaire et traversant en oblique toute la carapace pour aboutir au bord postéro-dorsal: cette carène est finement denticulée sur son premier tiers. Plus ventralement et postérieurement il existe deux petites carènes, parallèles au bord ventral (fig. $3 \mathrm{~B}, 3 \mathrm{C}$ ).

Les segments thoraciques sont libres, bien visibles et les deux derniers possèdent un petit prolongement pointu au bord postéro-ventral (fig. $3 \mathrm{C}$ ).

Les 4 premiers segments abdominaux sont courts, le 5ème est deux fois plus long que chacun des premiers et le Gème segment est le plus court de tous (fig. 3C).

Le telson est allongé, presque aussi long que le pédoncule des uropodes. II porte 3 épines apicales (une médiane longue et deux latérales courtes). Il porte aussi deux paires d'épines latérales situées sur le quart postérieur. Latéralement et sur presque toute sa longueur, on observe une rangée de fines denticulations (fig. 3J).

Le pédoncule des uropodes est deux fois plus long que les rames qui sont subégales. Le bord externe du pédoncule est bordé par une rangée de fines denticulations, rangée qui existe aussi sur le tiers postérieur du bord interne entre les 4 épines (fig. 3J).

Les deux rames de l'uropode ont la même taille et sont toutes deux bi-articulées. Elles portent une rangée de denticulations au bord interne.

La rame interne a le premier article armé de 3 épines et deux fois plus long

4) Cette espèce est respectueusement dédiée au Professeur Cl. Delamare Deboutteville. 


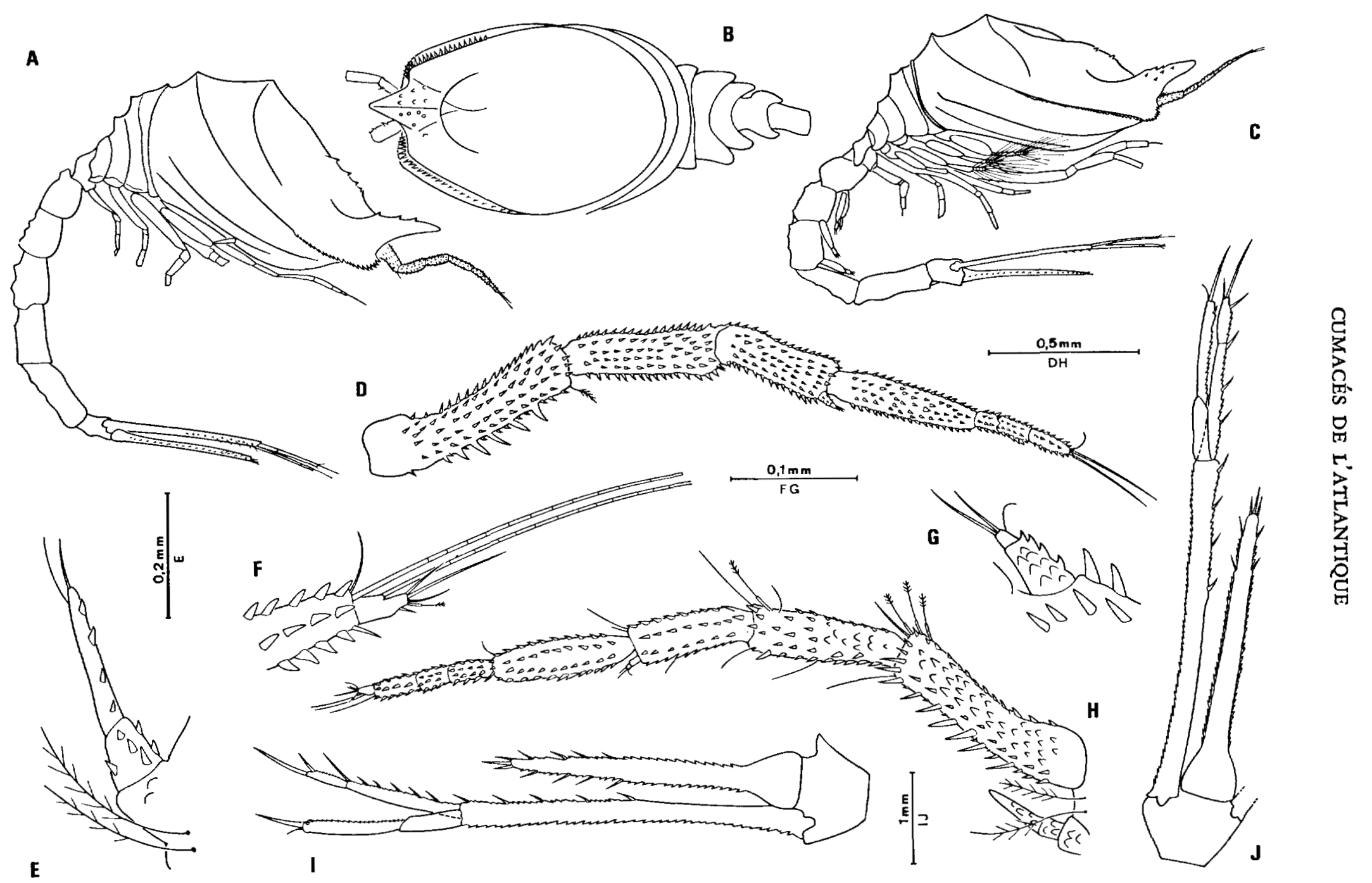

Fig. 3. Pseudodiastylis delamarei n. sp. A, habitus $\$ ; \mathrm{B}$, habitus $\delta$; C, habitus $\hat{o} ; \mathrm{D}, \mathrm{A1} \delta$; $\mathrm{E}$, $\mathrm{A2}$ \%; F, détail de l'extrémité de A1 $\$$; G, flagelle accessoire de A1 $A ; H, A 1$ et $\mathrm{A} 2$ \%; I, telson $\% ; \mathrm{J}$, telson $\hat{o}$. 
que le second. L'article distal porte une épine interne, une épine au bord distal externe et une longue épine apicale.

La rame externe a un premier article court, inerme et un second article deux fois plus long que le premier et portant une petite épine au bord distal interne, une soie externe et une longue épine apicale (fig. $3 \mathrm{~J}$ ).

L'antennule est caractéristique du genre, avec un pédoncule à trois articles de taille décroissante, recouverts de rangées longitudinales de dents. Le flagelle principal a 4 articles, le premier plus long que l'ensemble des 3 derniers et touts recouverts de rangées de dents (fig. 3D).

Le flagelle accessoire est très petit, composé d'un article basal massif portant quelques dents ou écailles, le second glabre (fig. 3G). Les pièces buccales sont banales pour un Lampropide. La MXP2 est massive, avec un basis aussi long que l'ensemble de tous les autres articles (fig. 4D). Les MXP3 ont le méropodite avec un prolongement distal et un exopodite bien développé (fig. 4C).

Les pattes thoraciques sont massives et courtes, les 4 premières paires avec un exopodite et les $\mathrm{P} 5$ sont présentes.

Les MXP3 et les pattes thoraciques sont toutes recouvertes de petites dents imbriquées en écailles (fig. $4 \mathrm{~A}, 4 \mathrm{E}, 4 \mathrm{~J}$, $4 \mathrm{~L}$ et $4 \mathrm{~K}$ ).

Il existe 3 paires de pléopodes, composées d'un premier article couvert de dents et d'écailles et de deux rames plus courtes que la moitié du premier article, la rame interne est égale à la moitié de l'externe, elle a deux articles de taille semblable et l'article distal porte 2 à 3 soies.

La rame externe n'a qu'un seul article et porte 3 soies distales et un petit prolongement latéral. Les deux rames sont dépourvues d'écailles ou de dents (fig. 4B).

Femelle. La forme générale est semblable à celle du mâle. Le pseudorostre est plus pointu, légèrement redressé vers le haut et il existe quelques dents plus marquées sur la ligne médiodorsale antérieure de la carapace et du pseudorostre (fig. 3A).

Le telson a la même forme que chez le mâle, mais porte 4 paires d'épines latérales. Le pédoncule des uropodes porte 5 épines internes et le premier article de la rame interne a 5 épines (fig. 3I).

L'antennule a la même structure que celle du mâle, elle porte de nombreuses soies sensorielles sur les bords distaux des deux premiers articles. Le flagelle principal possède un cinquième et dernier article de très petite taille qui n'existe pas chez le mâle, garni de soies sensorielles. Le flagelle accessoire plus petit encore que celui du mâle est dépourvu de dents ou d'écailles, alors que le pédoncule et le flagelle principal sont recouverts de rangées longitudinales de dents (fig. $3 \mathrm{H}, 3 \mathrm{~F})$.

L'antenne est très courte, plus petite que le premier article du pédoncule de l'antennule. Elle a trois articles, les deux premiers courts et massifs, le troisième plus long que les deux premiers réunis et portant quelques petites dents (fig. $3 \mathrm{H}, 3 \mathrm{E}$ ). 


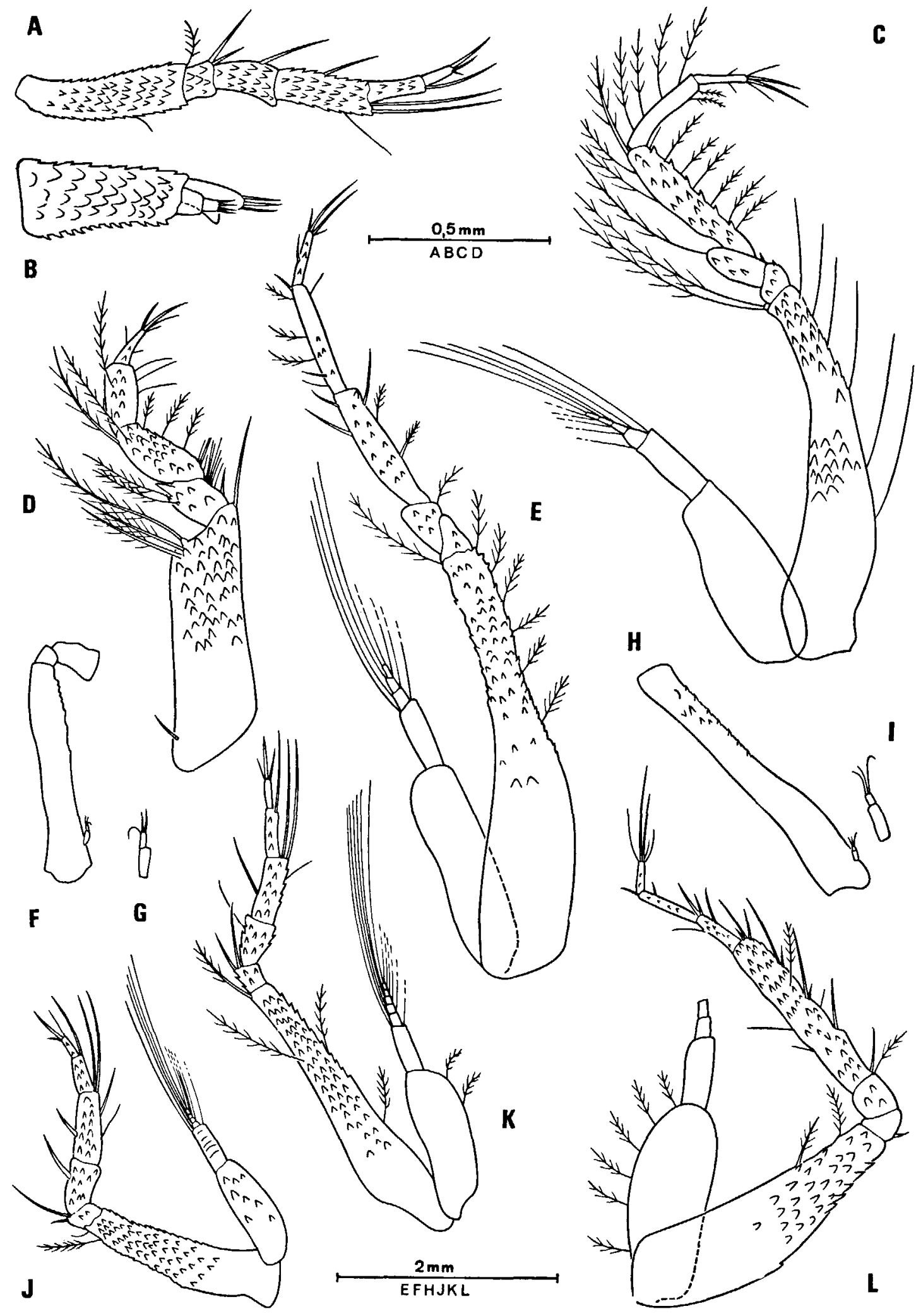

Fig. 4. Pseudodiastylis delamarei n. sp. A, P5; B, pléopode ô; C, MXP3 ô; D, MXP2 ô; E, P1 ô;

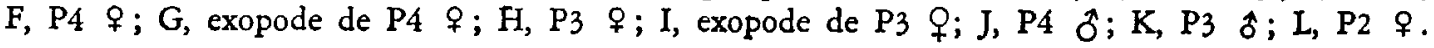


Les pièces buccales, les maxillipèdes et les pattes thoraciques sont semblables dans les deux sexes. Les MXP3, P1 et P2 ont des exopodites bien développés alors que les $\mathrm{P} 3$ et $\mathrm{P} 4$ possèdent des exopodites rudimentaires, très petits et biarticulés (fig. 4F, 4G, 4H et 4I).

Discussion. - Le genre Pseudodiastylis n'est connu que d'après une seule espèce, $P$. ferox Calman, 1905, récoltée à un seul exemplaire femelle par l'expédition de la "Siboga" au sud de la Malaisie, dans la mer de Banda, par $1158 \mathrm{~m}$ de profondeur.

La présence de trois paires de pléopodes chez le mâle, le premier segment de la rame externe de l'uropode beaucoup plus court que le segment distal, la présence d'une cinquième paire de pattes thoraciques, la présence d'exopodites rudimentaires sur les $\mathrm{P} 3$ et $\mathrm{P} 4$ de la femelle, le pseudorostre pointu et légèrement redressé et enfin le telson presque aussi long que le pédoncule des uropodes font de notre espèce un Pseudodiastylis indiscutable.

$P$. ferox est caractérisé par l'ornementation de la carapace, recouverte de nombreuses épines à la structure très particulière et par son pseudorostre aussi long que les $2 / 3$ de la carapace. Cette ornementation épineuse en fait une espèce très différente de $P$. delamarei $n$. sp. qui est caractérisée par les carènes de la carapace. Il existe cependant une ressemblance remarquable entre ces deux espèces, en particulier dans la structure et l'ornementation des antennules et des MXP3 (méropodite) des femelles.

Remarque. - Si l'on peut noter que Pseudodiastylis delamarei n. sp. a été récoltée dans l'Océan Atlantique à une latitude et une profondeur comparables à celles de la localité type de $P$. ferox en Malaisie, il est à peu près impossible de tirer d'autres conclusions d'ordre biogéographique de ces observations.

En effet l'état actuel des connaissances sur les faunes cumacéologiques dans l'hémisphère sud et dans les eaux tropicales est essentiellement limité à des eaux côtières de quelques secteurs géographiques: côtes d'Afrique du Sud, côtes d'Australie et de Nouvelle Zélande par exemple.

Les faunes profondes nous sont encore à 'peu près inconnues: il suffit, pour s'en persuader, de savoir que sur les quelques 104 espèces récoltées sur le transect Dakar-Recife (dont font partie les deux espèces étudiées dans ce travail), $73 \%$ sont nouvelles! (Jones \& Sanders, 1972). Ce chiffre permet d'imaginer ce que peut réserver l'étude des faunes profondes de l'Atlantique Sud et de l'Océan Indien.

\section{SUMMARY}

(1) Description of a new genus and new species of Diastylidae, Atlantistylis chauvini, from deep waters of the tropical Atlantic. This genus is characterized by having the telson shorter than the last somite, without lateral spines, whereas the inner ramus of the uropod of the male is twosegmented. The female has an exopod on the third maxilliped.

(2)Description of a new species of Pseudodiastylis (Lampropidae), P. delamarei, characterized by the presence of ridges on the carapace. 


\section{CUMACÉS DE L'ATLANTIQUE}

\section{REFERENCES}

BACEsCU-Mester, L., 1967. Contribution to the knowledge of the genus Leptostylis Sars (Cumacea): three new species collected by the Vema expedition. Crustaceana, 13: 265-275.

Calman, W. T., 1905. The Cumacea of the Siboga Expedition. Siboga-Exped. Monogr., 36: 1-23. - 1911. On new or rare Crustacea from the order Cumacea from the collection of the Copenhagen Museum, II. Trans. zool. Soc. London, 18: 311-398.

JoNEs, N. S., 1969. The systematics and distribution of Cumacea from depths exceeding 200 meters. Galathea Rep., 10: 99-178.

Jones, N. S. \& H. L. SANDers, 1972. Distribution of Cumacea in the Deep-Atlantic. Deep-Sea Res., 19 (10): 737-745.

Norman, A. M., 1879. Crustacea Cumacea of the "Lightning", "Porcupine" and "Valorous" Expeditions. An. Mag. nat. Hist., (5) 3: 54-73.

SARS, G. O., 1869. Undersøgelser over Christianiafjordens Dybvandfauna. Nyt Mag. Naturvid., 16: 1-344.

StebBing, T. R. R., 1910. The Sympoda, V. Ann. South Afr. Mus., 6 (4): 409-418.

Reçu le 28 mars 1973. 